\title{
Synthesis of Graphene Supported Nanocatalyst and Its Electrocatalytic Performance for Methanol Oxidation
}

\author{
Xinhua Lu \\ Nanjing Polytechnic Institute, Nanjing, Jiangsu, 210048
}

Keywords: Graphene Supported Nanocatalyst, Electrocatalytic Performance, Methanol Oxidatio

\begin{abstract}
The improved Hummers method was used to prepare the graphene oxide. Then, using the precursor as the carrier and the triblock copolymer P123 as the reductant, the protective agent and the morphology control agent, the liquid phase co-reduction method and the continuous reduction method were respectively used to prepare the graphene oxide Three graphene-supported Pt Pd (Pt Pd / G) nanocatalysts were synthesized. The graphene oxide and the metal precursor were simultaneously reduced to achieve the in-situ loading effect. The morphology, structure and composition of Pt Pd / G nanocatalyst were characterized by X-ray diffraction (XRD), transmission electron microscopy (TEM) and X-ray photoelectron spectroscopy (XPS). The results showed that both catalysts were nanodiesel structures. Hollow nanostructures were obtained by continuous reduction method. Electrochemical cyclic voltammetry and chronoamperometry showed that the Pt $\mathrm{Pd} / \mathrm{G}$ nanocatalysts with the hollow structure were the most resistant to CO poisoning and the Pt Pd / G nanocomposite catalysts synthesized at $100^{\circ} \mathrm{C}$ had the best electrocatalytic oxidation Methanol performance, about 1.5 times the commercial Pt / C catalyst.
\end{abstract}

\section{Introduction}

Direct methanol fuel cell (DMFC) has high energy density, low operating temperature, green pollution-free, wide range of fuel sources and is easy to store. It is a promising green energy source and has drawn wide attention from all walks of life. Precious metal Pt is widely used and difficult to replace catalyst in DMFC at present, but its reserves are small and expensive, which leads to its large-scale application in fuel cell field is hindered. At the same time, pure Pt catalyst is easily poisoned by intermediate of alcohol oxidation While reducing the catalytic activity and stability 1. Therefore, reducing the amount of Pt while increasing its catalytic activity and stability is the only way to achieve the commercialization of DMFC.

Graphene is one of the most promising carrier materials for many fuel cell catalyst carriers due to its good electrical and thermal conductivity, high strength and good flexibility, large specific surface area and easy modification. The lattice spacing between Pt and Pd is very close. Pd can weaken the force of Pt-COad to enhance the poisoning resistance of the catalyst. The price of Pd is only about $1 / 4$ of that of Pt. The addition of Pd can effectively reduce the amount of Pt Therefore, Pt Pd nanocatalysts are the hot topics of Pt-based fuel cell research. At present, scientists have done a lot of work on graphene-supported Pt-Pd nanocatalysts 27-29, in which a common method is to load graphene and a metal catalyst step by step, that is, to first reduce graphene oxide into a graphene carrier, The Pt Pd nanoparticles are reduced on graphene. The synthesis method has the disadvantages of complicated processes and high costs. Meanwhile, due to van der Waals forces, graphene oxide layers are easily accumulated during the reduction of graphene oxide into graphene, thereby greatly reducing the effective load area Resulting in subsequent Pt Pd nanoparticles can not be uniformly dispersed in the graphene, affecting the performance of the catalyst.

\section{Experimental Preparation and Implementation}

Potassium chloroplatinate (K2PtCl6, 99.9\%), potassium chlorodipalladate (K2PdCl4, 99.99\%), P123 and Nafion solution were all purchased from Sigma Aldrich; natural flake graphite The products were purchased from Zhongke Hengda Graphite Co., Ltd. Commercially available Pt / C 
catalyst (20\% (w)) was purchased from Alfa Aesar. The anhydrous methanol, concentrated H2SO4, NaNO3, $\mathrm{H} 2 \mathrm{O} 2$ and $\mathrm{KMnO} 4$ were all analytically pure. Water is double distilled water. X-ray powder diffraction (XRD) testing was performed on FEI Te GNai-F30 using a Rigaku Ultima IV $\mathrm{X}$-ray diffractometer, transmission electron microscopy (TEM) and high resolution transmission electron microscopy (HRTEM) using X-ray photoelectron spectroscopy (XPS), Thermogravimetric tests were performed on a STA 449 F5 Jupiter ${ }^{\circledR}$ thermogravimetric analyzer and the electrochemical test using the AUTOLAB (PGSTAT 12) electrochemical workstation.

The modified Hummers method 31 was used to prepare graphene oxide. The preparation method was as follows: $2.0 \mathrm{~g}$ of natural flake graphite and $1.0 \mathrm{~g}$ of NaNO3 were added into $40 \mathrm{ml}$ of concentrated sulfuric acid which had been precooled to $0^{\circ} \mathrm{C}$. After stirring for $0.5 \mathrm{~h}, 6.0 \mathrm{~g} \mathrm{KMn} \mathrm{O4}$ at $40^{\circ} \mathrm{C}$ for $2 \mathrm{~h}$, then slowly add $90 \mathrm{~m} \mathrm{~L}$ distilled water and react at $98^{\circ} \mathrm{C}$ for $0.5 \mathrm{~h}$, then add 300 $\mathrm{m} \mathrm{L}$ distilled water and $20 \mathrm{~m} \mathrm{~L} \mathrm{H} 2 \mathrm{O} 2$ to the mixture and stir for $0.5 \mathrm{~h}$ to The mixture turns bright yellow. Washed with $5 \% \mathrm{HCl}$ and distilled water until the filtrate was neutral, filtered, washed and dried to obtain graphite oxide, which was then configured as $1.0 \mathrm{mg} \cdot \mathrm{m} \mathrm{L}-1$ graphite oxide dispersion for later use.

$10 \square \mathrm{L} \mathrm{Pt} \mathrm{Pd} / \mathrm{G}$ ethanol dispersion $(2.0 \mathrm{mg} \cdot \mathrm{m} \mathrm{L}-1)$ on the polished glassy carbon electrode, after natural drying continue to drop $10 \square$ L of Nafion solution, after drying at room temperature work Electrode Pt Pd / G / GC. The theoretical loading of Pt on the electrode surface catalyst was $0.01 \mathrm{mg}$. Electrochemical performance test was carried out in a three-electrode system electrolytic cell. The auxiliary electrode was a platinum plate electrode and the reference electrode was a saturated calomel electrode (SCE). High purity nitrogen gas was removed for 15 min before electrochemical experiments to remove dissolved oxygen in the electrolyte. The CO dissolution test was performed in $0.1 \mathrm{~mol} \bullet \mathrm{L}-1 \mathrm{H} 2 \mathrm{SO} 4$ solution. The cyclic voltammetry and chronoamperometry of methanol were carried out in $0.1 \mathrm{~mol} \cdot \mathrm{L}-1 \mathrm{H} 2 \mathrm{SO} 4+0.5 \mathrm{~mol} \cdot \mathrm{L}-1 \mathrm{CH} 3 \mathrm{OH}$ solution.

Take $10 \mathrm{~m}$ L graphite oxide dispersion in a three-necked flask, ultrasonic stripping $1 \mathrm{~h}$ get graphene oxide dispersion, add 1.0 g P123, stirred and sonicated to dissolve, magnetic stirring and control the temperature at $30^{\circ} \mathrm{C}$, using a total of Reduction method, $10 \mathrm{~m} \mathrm{~L} \mathrm{K2Pt} \mathrm{Cl6} \mathrm{solution}$ (0.03 mol $\bullet \mathrm{L}-1)$ and $10 \mathrm{~mL} \mathrm{K2PdCl4}$ solution (0.03 mol $\bullet$ L-1) were added dropwise to the three-necked flask with a constant pressure funnel at the same time. The reaction was carried out for $2 \mathrm{~h}$, centrifuged and washed three times to obtain Pt Pd / G-1 and dispersed in anhydrous ethanol. The reaction temperature was kept at $100^{\circ} \mathrm{C}$. and the other reaction conditions were kept unchanged. The sample was labeled Pt Pd / G-2 ; The same reaction temperature at $100{ }^{\circ} \mathrm{C}$, the amount of the reaction remained unchanged by continuous reduction method, that is, with a constant pressure funnel K2Pd Cl4 solution was slowly added dropwise after $2 \mathrm{~h} \mathrm{K2Pt} \mathrm{Cl6} \mathrm{solution}$ was added dropwise and the reaction was continued for $2 \mathrm{~h}$, This method gives the sample labeled Pt Pd / G-3.

\section{Results and discussion}

The XRD patterns of graphene (G-100) prepared by reduction of graphite, graphite oxide (GO), $\mathrm{P} 123$ reduced at $30^{\circ} \mathrm{C}$ and $\mathrm{G}-100$ obtained at $100^{\circ} \mathrm{C}$ were obtained. It is observed that the obvious diffraction peak appears at $26.3^{\circ}$, which is the characteristic diffraction peak of the natural graphite (002) crystal plane, and the peak intensity is relatively large. After the strong oxidant, the graphite is oxidized to graphite oxide, and the characteristic diffraction peak of the (002) Shifted to the left, and the diffraction peak of the (002) plane of graphite oxide appeared at $11.1^{\circ}$. The graphene oxide after the stripping was changed to graphene oxide. After reduction by P123 at $30^{\circ} \mathrm{C}$ and $100{ }^{\circ} \mathrm{C}$, G- 30 and G-100. Both of them show obvious peaks near $24.5^{\circ}$, which is characteristic diffraction peak of (002) plane of graphene, which indicates that graphene oxide can be reduced by P123 to obtain reduced graphene oxide. Figure 1 (b) compares XRD patterns of Pt Pd / G-1, Pt Pd / G-2 and Pt Pd / G-3 with Pt Pd appearing around $39^{\circ}, 45^{\circ}, 66^{\circ}, 80^{\circ}$ and $84^{\circ}$ The diffraction peaks of (111), (200), (220), (311) and (222) crystal planes are proved to have a face-centered cubic structure. The broad peaks around $24^{\circ}$ are diffraction peaks of (002). Figure 1 (c) is a TEM image of graphite oxide. As can be seen from the figure, the prepared graphite oxide is a transparent sheet 
structure, and at the same time can observe obvious wrinkle layers, which can provide larger load area. Low TEM image 2 (a1-c1) shows that the Pt Pd nanoparticles in the three kinds of Pt Pd / G are well dispersed and supported on the surface of the graphene. The inset in Fig. 2 (a1-c1) is a selective region electron diffraction (SAED) diagram, with four distinct diffraction rings corresponding internally to the four planes (111), (200) (220), (311), which corresponds to the first four distinct diffraction peaks in the XRD spectrum. The results showed that Pt Pd / G-1 and Pt Pd / G-2 with nanowire structures obtained by co-reduction method showed average diameters of $19 \mathrm{~nm}$ and $23 \mathrm{~nm}$, respectively. The Pt Pd / G-2 synthesized by the continuous reduction method is an irregular-shaped hollow structure with an average particle diameter of $13 \mathrm{~nm}$, and at the same time, particles of Pt Pd nano particles with smaller particle sizes can be observed. Figure 2 (a3-c3) can be more intuitive to see the fine structure of the three kinds of nano-catalysts: Pt Pd / G-1 (a3), Pt Pd / G-2 The darker outline of the nanostructures in Pt Pd / G-3 (c3) confirmed its hollow structure. The inset in Figure 2 (a3-c3) is an enlarged view of the white box area, with clear lattice fringes clearly visible, with lattice spacings around $0.23 \mathrm{~nm}$, Face-centered cubic structure Pt Figure 1 (a) Graphite, graphite oxide (GO), graphene obtained by reduction of $\mathrm{P} 123$ at $30^{\circ} \mathrm{C}(\mathrm{G}-30)$, graphene reduced by P123 at $100^{\circ} \mathrm{C}$ (B) XRD patterns of Pt Pd / G-1, Pt Pd / G-2 and Pt Pd / G-3; (c) lattice spacing of the (111) crystal plane Match.

The nanodial structures of Pt Pd / G-1 and Pt Pd / G-2 were prepared under one-step co-reduction conditions. It was found that triblock copolymer P123 consisted of hydrophilic PEO and hydrophobic PPO with Reducing PEO fragments can reduce Pd precursors at room temperature but not Pt precursors, both of which can be reduced at high temperatures. The special structure and selective reduction of Pt and Pd at low temperatures enable P123 As a good topography control agent 18,32. Under the condition of continuous reduction at $100^{\circ} \mathrm{C}$, the $\mathrm{Pd}$ precursor is first completely reduced to $\mathrm{Pd}$ and then added to the Pt precursor. Since the reduction potential of $\mathrm{Pt}$ is higher than the reduction potential of $\mathrm{Pd}$, the Pd element can undergo a potential substitution reaction with the Pt precursor, So as to obtain a hollow structure 33. In the TEM image, we can find that some of the smaller nanoparticles that do not form a hollow structure may be due to the reduction of part of the Pt precursor directly by P123, and some grow on the surface of the Pd nanoparticle to form Pt Pd core-shell structure. To this end, we try to use continuous reduction method at $30^{\circ} \mathrm{C}$ in an attempt to avoid direct reduction of Pt precursors at high temperatures, but repeated tests show that the synthesized materials exhibit poor electrocatalytic oxidation of methanol under acidic conditions, However, it has some catalytic activity under alkaline conditions. Therefore, we speculate that during the continuous reduction process at low temperature, it may be due to the interaction between graphene and Pt precursor that the nucleated Pd can not react with the Pt precursor Displacement reaction. The three kinds of Pt Pd / G are all precursors of graphene oxide and metal precursors during the synthesis process. The previous studies showed that graphene oxide had defects in the reduction process of P123. The reduced Pt and Pd precursors Experience preferred nucleation and growth defects in order to achieve the effect of in situ load, which makes the metal particles and graphene stronger force will not easily fall off, but also makes the metal particles loaded on the graphene More uniform.

Chronoamperometry is an important means to evaluate the electrocatalytic oxidation of methanol to methanol. The chronoamperometry curves of four catalysts at $0.6 \mathrm{~V}$ potentials in $0.1 \mathrm{~mol} \cdot \mathrm{L}-1$ $\mathrm{H} 2 \mathrm{SO} 4+0.5 \mathrm{~mol} \cdot \mathrm{L}-1 \mathrm{CH} 3 \mathrm{OH}$ solution . It can be observed that the catalyst will generate a larger initial current in the initial stage of the test, then the speed decreases and tends to be stable. This is due to the step-by-step overpotential momentarily produced when the potential reaches the set constant potential of $0.6 \mathrm{~V}$ during the chrono-current test, resulting in a significantly larger transient current which decays rapidly over time, indicating In addition, when the electrode potential changes instantaneously, it takes a certain amount of power to charge the electric double layer. Due to the large electric double layer of graphene, the relative commercial $\mathrm{Pt} / \mathrm{C}$, the starting current is significantly higher than Pt / C. The combined effect of the two results in a much higher initial current density of Pt Pd / G in the J-t curve than the theoretical value. The stability of the current density from descending order: Pt Pd / G-2 $\square$ Pt Pd / G-3 $\square$ Pt / C $\square$ Pt Pd / G-1, and sulfuric acid 
in methanol solution positive sweep peak current density Trend consistent. The results of chronoamperometry and cyclic voltammetry show that Pt Pd / G-2 has higher catalytic activity and stability to methanol oxidation.

\section{Conclusion}

Three kinds of Pt Pd / G nanocatalysts were prepared by liquid co-reduction method and continuous reduction method. The precursor of the graphene oxide was reduced synchronously with the metal precursor to achieve the in-situ loading of graphene. XRD, TEM and XPS characterization analysis showed that graphene oxide was reduced to graphene. Pt Pd / G-1 and Pt Pd / G-2 catalysts obtained by the co-reduction method were obtained by the continuous reduction method Pt Pd / G-3 hollow structure. Electrochemical tests showed that Pt Pd / G-3 possesses good resistance to CO poisoning and Pt Pd / G-2 electrocatalytic oxidation of methanol.

\section{References}

[1] Inner Mongolia to create China's first comprehensive graphene materials Institute [J]. China Powder Industry. 2013 (04)

[2] Liu Tong, Zhang Jinqiu.Catalytic performance of in-situ electrodeposited AuPt alloys on three kinds of carbon materials [J] .Chinese Journal of Inorganic Chemistry. 2017

[3] Zhang Min, Shang Xiaopeng.Application of Graphene and Its Composites in Electrochemistry [J]. Journal of Colloid and Polymer. 2012 (04)

[4] Magical graphene [J]. Taizhou Technology. 2011 (09)

[5] Semiconductor Institute made new progress in the study of the physical properties of multilayer graphene [J]. China Powder Industry. 2012 (02)

[6] Wu Yuzhu, Wu Xiangfeng. Research progress of graphene [J]. China Powder Industry. 2013 (04) 